\title{
PC Program for Obtaining Orthogonal Polynomial Regression Coefficients for Use in Longitudinal Data Analysis
}

\author{
THOMAS R. 'TEN HAVE', CHARLES J. KOWALSKI ${ }^{2}$, AND \\ EMET D. SCHNEIDERMAN'3 \\ ${ }^{7}$ Department of Binstatistics and ${ }^{2}$ Department of Oral Biology, The \\ University of Michigan, Ann Arbor, Michigan $48109 ;{ }^{3}$ Department of \\ Oral and Maxillofacial Surgery, Baylor College of Dentistry, Dallas, \\ Texas 75246
}

\begin{abstract}
Much of longitudinal data analysis begins with dimensionality reduction, i.e., the replacement of the $\mathrm{T}$ observations $\mathrm{x}_{1}, \mathrm{x}_{2}, \ldots, \mathrm{x}_{\mathrm{T}}$ on an individual taken at times $t_{1}, t_{2}, \ldots, t_{T}$ (not necessarily equally spaced) by a smaller number, $P$, of parameters which are then used to describe and compare growth processes. We focus on the class of polynomial growth curve models for one-sample data matrices in which the $P$ regression coefficients are estimated by an equation of the form $\hat{\tau}=\left(W^{\prime} W{ }^{1} W^{\prime} x\right.$ and consider the choice of the design matrix $W$. The case in favor of using orthogonal polynomials to comprise the elements of $W$ and provide a PC program, written in GAUSS, for obtaining them is presented. This program can be used instead of existing tables of orthogonal polynomials in the case of equally spaced time points, and to avoid laborious hand-computation to obtain them when the time points are not equally spaced. The program also computes the corresponding orthogonal polynomial regression coefficients $\hat{\alpha}=\left(\Phi^{\prime} \Phi\right)^{-1} \Phi^{\prime} \mathbf{x}$, where $\Phi$ consists of orthogonal polynomials, which may then be input into other programs for subsequent analysis, e.g., to compare the growth profiles of several groups of individuals. Examples of the use of the program are given. Information on obtaining a copy of the program is provided in Appendix A.
\end{abstract}

1992 Wiley-Liss, Inc.

Longitudinal data analysis is concerned with data sets of the form

$$
\underset{\mathrm{N} \times \mathrm{T}}{\mathbf{x}}=\left[\begin{array}{cccc}
\mathrm{x}_{11} & \mathbf{x}_{12} & \cdots & \mathrm{x}_{1 \mathrm{~T}} \\
\mathrm{x}_{21} & \mathrm{x}_{22} & \cdots & \mathbf{x}_{2 \mathrm{~T}} \\
\vdots & \vdots & \cdots & \vdots \\
\mathbf{x}_{\mathrm{N} 1} & \mathbf{x}_{\mathrm{N} 2} & \cdots & \mathrm{x}_{\mathrm{NT}}
\end{array}\right] \rightarrow\left[\begin{array}{c}
\mathbf{x}_{1}^{\prime} \\
\mathbf{x}_{2}^{\prime} \\
\vdots \\
\mathrm{x}_{\mathrm{N}}^{\prime}
\end{array}\right](\mathbf{1})
$$

where $x_{i j}$ is the value of the measurement made on individual $i(i=1,2, \ldots, N)$ at time $t_{j}(j=1,2, \ldots, T)$ and $x_{i}$ is the $T \times 1$ vector of observations for the $i^{t h}$ individual. Often a group structure is imposed on $\mathrm{X}$, e.g., the $\mathrm{N}$ individuals may belong to $G$ different groups, the $\mathrm{g}^{\text {th }}$ group comprising $\mathbf{n}_{\mathrm{g}}$ members with $\sum_{\mathrm{g}=1}^{\mathrm{G}} \mathrm{n}_{\mathrm{g}}=\mathrm{N}$, and the primary aim of the analysis is to test for differences between the growth patterns exhibited by these groups.

A wide variety of approaches to the analysis of such data sets have been developed (see
Goldstein, 1979; or reviews by Kowalski and Guire, 1974; Guire and Kowalski, 1979), many of which seek to reduce the dimensionality of the problem by replacing the $T$ observations on each individual by a smaller number, $P$, of parameters which retain most of the information contained in the course of growth actually observed. Examples of this strategy include the use of principal components analysis (Rao, 1958; Church, 1966), tracking indices (McMahan, 1981), and the fitting of polynomials to the individual growth profiles as originally proposed by Wishart (1938). For a good review, see Marubini and Milani (1986).

It seems likely that the strategy of dimensionality reduction was motivated by computational considerations and/or to "achieve parsimony," but this approach may also be defended on the grounds of power. By fitting a model with fewer parameters than the

Received August 15, 1990; accepted September 9, 1991

Address reprint requests to Dr. Emet D. Schneiderman, Baylor College of Dentistry, 3302 Gaston Ave., Dallas, TX 75246. 
number of time points $\mathrm{T}$ and then performing a multivariate analysis of variance (MANOVA) on estimates of these parameters, one can expect increased power over simply performing the MANOVA on the original ' $\mathrm{T}$ measurements (Das Gupta and Perlman, 1974; Allen, 1983). Moreover, to the extent that these parameters are biologically meaningful, the interpretation of any differences found may be facilitated (Kowalski, 1972).

Perhaps the most widely used technique for dimensionality reduction in the context of growth curve analysis involves replacing the observations $x_{i}$ by estimates of the regression coefficients obtained by fitting a polynomial to these observations. Following the notation established in Schneiderman and Kowalski (1985, 1989; Schneiderman et al., 1990 ), we denote the $P \times 1$ vector of estimated regression coefficients for the $i^{t h}$ individual, obtained by fitting a polynomial of degree $\mathrm{D}=\mathrm{P}-1$ to $\mathrm{x}_{\mathrm{i}}$, by $\hat{\tau}_{i}$; and the $\mathrm{N} \times \mathrm{P}$ matrix containing the values of these coefficients for each of the $\mathrm{N}$ individuals by $\tau$. The dimension reduction strategy in this case is then accomplished by replacing $\underset{\mathrm{NxT}}{\mathrm{X}}$ by ${ }_{\mathrm{N}}^{\stackrel{+}{\mathrm{T}} \mathrm{P}}$. Instead of performing the MANOVA on the data points $\mathrm{X}$, we do the MANOVA on the regression coefficients $\hat{\tau}$ and can thereby expect more sensitive tests and more readily interpretable results. The question remains as to how to estimate the $\tau_{\mathrm{j}}$. The process and the model underlying it are described below.

\section{ESTIMATION OF}

If we assume that the $\mathbf{x}_{\mathrm{i}}$ have multivariate normal distributions with mean or expected values

$$
\mathrm{E}\left(\mathbf{x}_{\mathbf{i}}\right)=\mathrm{W} \tau_{\mathbf{i}}
$$

and common covariance matrix $\Sigma$, then the best linear unbiased estimator (BLUE) of $\tau_{i}$ is (Draper and Smith, 1966, p. 77)

$$
\tau_{\mathrm{i}}=\left(\mathbf{W}^{\prime} \Sigma^{-1} \mathbf{W}\right)^{-1} \mathbf{W}^{\prime} \Sigma^{-1} \mathbf{x}_{\mathrm{i}}
$$

In the above, $W$ is a $T \times P$ matrix of known constants, the so-called within-individual or time design matrix (Schneiderman and Kowalski, 1985).

Since $\Sigma$ is in general unknown, the problem of obtaining the BLUE of $\tau_{i}$ involves the question of what constitutes a suitable "substitute" for $\Sigma$. One of the more obvious choices is $\mathbf{S}$, the sample covariance matrix.
This was used by Rao (1959) and implemented in SAS (Schneiderman and Kowalski, 1985) and in GAUSS (Schneiderman and Kowalski, 1989). Potthoff and Roy (1964) recognized the arbitrary nature of this choice and developed the theory necessary to accommodate the situation in which $\Sigma$ is indeed replaced by an arbitrary (positivedefinite and symmetric) $\mathrm{T} \times \mathrm{T}$ matrix $\mathrm{A}$. They considered that the choices $\mathbf{A}=\mathbf{S}$ and $\mathbf{A}=\mathbf{I}$, the identity matrix, had certain advantages, but emphasized that their approach remained valid for any (positivedefinite and symmetric) matrix A. They gave an example using

$$
\mathrm{A}=\frac{1}{1-\rho^{2}}\left[\begin{array}{lllll}
1 & \rho & \rho^{2} & \cdots & \rho^{\mathrm{T}-1} \\
\rho & 1 & \rho & \cdots & \rho^{\mathrm{T}-2} \\
\vdots & \vdots & \vdots & \cdots & \vdots \\
\rho^{\mathrm{T}-1} & \rho^{\mathrm{T}-2} & \rho^{\mathrm{T}-3} & \cdots & 1
\end{array}\right]
$$

where they estimated $\rho$, the correlation between successive observations, on the basis of an independent experiment. An SAS program implementing their technique and allowing the use of $\mathbf{S}$, $\mathbf{I}$, or a user-defined $\mathbf{A}$ was developed by Ten Have et al. (1992). This program can also accommodate other special covariance structures, e.g., those considered by Morrison (1972), through an appropriate choice of $\mathrm{A}$.

More recently, attention has focused on the employment of models assuming patterns for $\Sigma$ for which (3) reduces to the simple (unweighted) form

$$
\hat{\tau}=\left(\mathbf{W}^{\prime} \mathbf{W}\right)^{-1} \mathbf{W}^{\prime} \mathbf{x}_{\mathbf{i}} .
$$

This occurs in the Potthoff and Roy analysis when $\mathrm{A}=\mathrm{I}$ and in the so-called random coefficients or two-stage polynomial growth curve model introduced by Rao (1965a) and subsequently developed and applied by Fearn $(1975,1977)$, McMahan (1981), Ware and Wu (1981), and Ware (1983). A GAUSS program for analyzing longitudinal data sets conforming to the two-stage model is also available (Ten Have et al., 1991). This model assumes that $\Sigma$ has the form

$$
\Sigma=\mathbf{W} \Lambda \mathbf{W}^{\prime}+\sigma^{2} \mathbf{I}
$$

and, when this assumption is tenable, Chinchilli and Carter (1984) have documented that the unweighted estimator (5) has distinct advantages. They also developed an 
SAS program to perform a likelihood ratio test for the goodness-of-fit of (6). Other examples of the use of the unweighted estimator (5) in longitudinal data analysis include Wishart (1938), Zerbe and Walker (1977), Zerbe (1979), and Allen (1983).

It is of considerable interest, then, to consider the use of $(5)$ in the study of growth and development and, in particular, the choice of $\mathrm{W}$ in the underlying model (2). While a number of investigators (e.g., Wishart, 1938; Rao, 1959; Zerbe and Walker, 1977; Goldstein, 1979; Zerbe, 1979; Allen, 1983) have used

$$
\mathbf{W}=\left[\begin{array}{cccc}
1 & t_{1} & \cdots & t_{1}^{\mathrm{D}} \\
1 & \mathrm{t}_{2} & \cdots & \mathrm{t}_{2}^{\mathrm{D}} \\
\vdots & \vdots & \cdots & \vdots \\
1 & \mathrm{t}_{\mathrm{T}} & \cdots & \mathrm{t}_{\mathrm{T}}^{\mathrm{D}}
\end{array}\right]
$$

Others (e.g., Rao, 1965a; Grizzle and Allen, 1969; McMahan, 1981; Ware and Wu, 1981) have employed orthogonal polynomial regression coefficients (OPRC's). The remainder of this paper discusses the use of OPRC's in longitudinal data analysis and provides a GAUSS program for generating them. We will document that OPRC's have definite advantages, not only with respect to computational speed and accuracy (which is relatively well-known), but also with regard to inference (which appears to be much less well known). We show that the optimality of OPRC's in ordinary least squares regression in the sense established by Rao (1965b, p. 194) extends to the two-stage model and, by means of an example, that more "sensible" conclusions concerning the growth curves in the groups under consideration may be drawn if OPRC's are used in place of $W$ as given in (7) to estimate the regression coefficients. We begin by indicating how the use of OPRC's originated and then proceed to consider their advantages. In what follows, W will always refer to the matrix defined by $(7)$. $\Phi$ (defined below) will contain the values of the orthogonal polynomials used to obtain the OPRC's.

\section{ORTHOGONAL POLYNOMIALS}

Notice that to compute (5), the $\mathrm{P} \times \mathrm{P}$ matrix $W^{\prime} W$ must be inverted, which, if the degree of the polynomial being fit is moderate to large, may involve considerable computational labor. Before the advent of modern computers it was obviously of inter- est to circumvent this problem and one of the most useful devices in this regard proved to be the use of orthogonal polynomials. The basic idea is to rewrite (2) in the form

$$
\mathbf{E}\left(\mathbf{x}_{\mathbf{i}}\right)=\Phi \alpha_{\mathbf{i}}
$$

$$
\text { where } \Phi=\left[\begin{array}{cccc}
1 & \phi_{1}\left(t_{1}\right) & \cdots & \phi_{\mathrm{D}}\left(\mathrm{t}_{1}\right) \\
1 & \phi_{1}\left(\mathrm{t}_{2}\right) & \cdots & \phi_{\mathrm{D}}\left(\mathrm{t}_{2}\right) \\
\vdots & \vdots & \cdots & \vdots \\
1 & \phi_{1}\left(\mathrm{t}_{\mathrm{T}}\right) & \cdots & \phi_{\mathrm{D}}\left(\mathrm{t}_{\mathrm{T}}\right)
\end{array}\right]
$$

and $\phi_{d}$ is a polynomial of degree $d$ with the properties

and

$$
\sum_{j=1}^{T} \phi_{d}\left(t_{j}\right)=0
$$

$$
\sum_{j=1}^{T} \phi_{d}\left(\mathbf{t}_{j}\right) \phi_{k}\left(\mathbf{t}_{j}\right)=0
$$

whenever $d \neq k$, i.e., $\phi_{1}, \phi_{2}, \ldots, \phi_{\mathrm{D}}$ are orthogonal. The $\alpha_{\mathrm{i}}$ in $(8)$ are the OPRC's and they are estimated as in (5), viz.,

$$
\hat{\alpha}_{\mathbf{i}}=\left(\Phi^{\prime} \Phi\right)^{-1} \Phi^{\prime} \mathbf{x}_{\mathbf{i}} .
$$

However, (12) is much easier to work with than is (5) because $\Phi^{\prime} \Phi$ is a diagonal matrix (by the definition of matrix multiplication and condition (11)) and the inverse of a diagonal matrix is obtained simply by taking the reciprocals of the diagonal elements of the matrix being inverted. One can even go a step further in this direction by using orthonormal polynomials, i.e., polynomials satisfying (10) and (11) and

$$
\sum_{j=1}^{T} \phi_{d}^{2}\left(\mathbf{t}_{j}\right)=1
$$

because then $\Phi^{\prime} \Phi=I$ and (12) reduces to

$$
\hat{\alpha}_{\mathbf{i}}=\Phi^{\prime} \mathbf{x}_{\mathbf{i}}
$$

It should be noted that it is always possible to rewrite $\mathrm{E}\left(\mathbf{x}_{\mathbf{i}}\right)=\mathbf{W} \tau_{\mathrm{i}}$ in the form $\Phi \alpha_{\mathbf{i}}$ where $\Phi$ contains the values of either orthogonal or orthonormal polynomials, and that from the 
computational point of view there are obvious advantages in replacing the $\mathrm{T}$ observations $\mathbf{x}_{\mathrm{i}}$ on individual $\mathbf{i}$ by $\hat{\alpha}_{i}$ (as opposed to $\hat{\tau}_{i}$ ) computed by using either (12) or (14). The question remains, however, as to whether replacing $W$ by $\Phi$ is "necessary," given that computers will be utilized to perform the computations. The case for an affirmative answer is summarized below. Here and in what follows no real distinction is made between the $\hat{\alpha}$ 's computed from (12) and those computed from (14). We refer to both as OPRC's and the users of our program will have the option of obtaining either set of coefficients (normalized or not).

I. If $\mathbf{W}^{\prime} \mathbf{W}$ is ill-conditioned (Bellman, 1970; Stewart, 1985), the use of (5) may be inaccurate. Small changes in $\mathbf{x}_{\mathbf{i}}$ may produce large changes in $\hat{\tau}_{\mathbf{i}}$. Equations (12) and/or (14) may have had their roots in computational speed but there presently exist situations in which accuracy is the more important consideration. Citing Bright and Dawkins (1965), Draper and Smith (1966, p. 152) concluded that, "Although orthogonal polynomials are often recommended only when desk calculators are used ... even when a computer is available, and especially when the polynomial is of high order, orthogonal polynomials are worthwhile. Using them provides greater computing accuracy and reduced computing times." The question of accuracy is of increasing importance the more the elements of $\mathbf{W}^{\prime} \mathbf{W}$ differ in magnitude (Neter et al., 1985), a condition that can occur for values of $t_{1}, t_{2}, \ldots, t_{T}$ commonly encountered in practice. Bock $(1979$, p. 211) also discussed the problem of accuracy and showed how the use of OPRC's circumvents all difficulties, concluding that, "the problem of round-off error, which plagues leastsquares fitting of higher-order polynomials is completely solved if the orthogonal polynomials are used."

II. When attempting to determine the lowest-degree polynomial adequate to fit the data, it is common practice to use step-up goodness-of-fit tests, i.e., to test $\mathrm{D}=1$, $\mathrm{D}=2$, etc., in turn until an acceptable fit is achieved. If (5) is used, the coefficients in $\hat{\tau}_{i}$ will change at each step (and $\left(\mathbf{W}^{\prime} \mathbf{W}\right)^{-1}$ needs to be recomputed each time), but if (12) or (14) is used the previously computed elements of $\hat{\alpha}_{i}$ are unaffected when $D$ is increased (Draper and Smith, 1966). And this is more than just of computational significance. Two additional, more substantive ad- vantages will be mentioned. The first is somewhat theoretical in nature, the second addresses an important practical problem.

The more theoretical aspect involves the facts that the estimated regression coefficients will be unbiased, $\mathrm{E}(\hat{\tau})=\tau$, only if the postulated model is correct (in particular, the degree of the polynomial used); and the extent of the bias encountered when an inappropriate model is used depends on the values of the elements of $W$ (Draper and Smith, 1966). Suppose, e.g., that $\mathrm{E}(\mathbf{x})=\mathrm{W}_{\boldsymbol{T}}$ is postulated but the true model is $\mathrm{E}(\mathbf{x})=\mathbf{W}_{\tau}+\mathbf{W}^{*} \tau^{*}$. Then $\mathrm{E}(\tau)=\tau+\mathbf{A} \tau^{*}$ where $A=\left(W^{\prime} W\right)^{-1} W^{\prime} W^{*}$ is called the alias matrix. This can be substantial if $W$ is used, but $\mathbf{A}=0$ when $\mathbf{W}=\Phi$. Draper and Smith (1966) give examples where a line $(D=1)$ is assumed, but the true model is (a) quadratic $(\mathrm{D}=2)$ and $(\mathrm{b})$ cubic $(\mathrm{D}=3)$. In both cases the $\mathrm{T}=7$ centered time points $-3,-2,-1,0$, $1,2,3$ were used in W. In (a),

$$
\begin{gathered}
\mathbf{E}\left(\hat{\tau}_{1}\right)=\tau_{1}+\frac{2}{3} \tau_{2} \\
\mathbf{E}\left(\hat{\tau}_{2}\right)=\tau_{2}
\end{gathered}
$$

so $\hat{\tau}_{1}$ is biased, $\hat{\tau}_{2}$ unbiased. In (b),

$$
\begin{aligned}
& \mathrm{E}\left(\hat{\tau}_{1}\right)=\tau_{1}+4 \tau_{2} \\
& \mathrm{E}\left(\hat{\tau}_{2}\right)=\tau_{2}+7 \tau_{3}
\end{aligned}
$$

so both estimates are biased. The extent of this bias will in general be even greater if $t_{1}$, $t_{2}, \ldots, t_{T}$ are used in $W$ without centering. What this means is that if too-low-a-degree polynomial is used in conjunction with W (whether the time points are centered or not), our estimate of $\tau$ can be substantially biased. No bias occurs when $\Phi$ is used. While tests for the adequacy of a given degree polynomial exist (e.g., Schneiderman and Kowalski, 1985), such tests are, of course, fallible.

The second point involves a more practical concern. Many, if not all, of the available methods for polynomial growth curve analysis require that polynomials of the same degree be fit to each individual in the sample. When, as is often the case, step-up goodness-of-fit tests are used to fit the individual growth curves prior to the analysis, one finds $\mathrm{D}_{\text {max }}$ and then either refits everyone to this degree (Dawson et al., 1980) or augments the vector of previously estimated regression coefficients with 0's (Zerbe, 1979). Little is 
known about which of these strategies is "best," but it should be noted that the use of orthogonal polynomials will minimize the differences between them, differences which can impact on the results of the analysis. Suppose, for example, that a line suffices to fit the data for a given individual, but $\mathrm{D}_{\max }=2$. If $\mathrm{W}$ is used the choice is between

$$
\left[\begin{array}{c}
\hat{\tau}_{1} \\
\hat{\tau}_{2}
\end{array}\right] \rightarrow\left[\begin{array}{c}
* \\
\tau_{1} \\
\tau_{2}^{*} \\
\tau_{3}^{*}
\end{array}\right] \text { or }\left[\begin{array}{c}
\tau_{1} \\
\tau_{2} \\
0
\end{array}\right]
$$

where the $*$ 's indicate the refitted values. These may differ appreciably from the estimates based on $\mathrm{D}=1$ (viz., $\hat{\tau}_{1}$ and $\hat{\tau}_{2}$ ). For example, consider the last subject (who is in no way "special") in the data set analyzed by Dawson et al. (1980). $D_{\max }$ was found to equal 2 for this data set, but a line fit this individual's growth profile adequately. For the line the estimated regression coefficients are $[43.551,0.749]^{\prime}$. When this individual's data are refit to $D_{\max }=2$, these coefficients are $[36.566,2.168,-0.070]^{\prime}$ and it is seen that the first two coefficients change dramatically. The choice is between using $[36.566,2.168,-0.070]^{\prime}$ and $[43.551,0.749$, $0.00]^{\prime}$ as the basic datum for this individual; and for many purposes this choice will be important.

On the other hand, if $\Phi$ is used, the choice is between

$$
\left[\begin{array}{c}
\hat{\alpha}_{0} \\
\alpha_{1}
\end{array}\right] \rightarrow\left[\begin{array}{c}
\alpha_{0} \\
\alpha_{1} \\
\alpha_{2}
\end{array}\right] \text { or }\left[\begin{array}{c}
\alpha_{0} \\
\alpha_{1} \\
0
\end{array}\right]
$$

and, since $\alpha_{2}$ will be small (and, as noted earlier, $\hat{\alpha}_{0}$ and $\hat{\alpha}_{1}$ do not change), the choice is all but moot. Note that while the elements of $\hat{\tau}$ are subscripted $1,2,3, \ldots$ we have followed the standard practice of subscripting the OPRC's by $0,1,2 \ldots$ The relationship between these two sets of coefficients is made clear in equations (16) and (17).

III. The use of OPRC's has a long history in longitudinal data analysis. Since formulae in many (current) publications are given in terms of OPRC's, practical application of these methods is facilitated when the coefficients are in fact employed by potential users. To cite but two examples, both Ware and
Wu (1981) and McMahan (1981) give their respective formulae for prediction intervals and tracking indices assuming $\mathbf{W}^{\prime} \mathbf{W}=\mathbf{I}$, i.e., they take $\mathbf{W}=\Phi$.

IV. When testing hypotheses concerning growth curves in several distinct groups of individuals, multivariate tests (e.g., MANOVA) based on the $\hat{\tau}_{i}$ will produce the same results ( $P$ values) as when the $\hat{\alpha}_{i}$ are used (i.e., the multivariate tests are invariant). However, univariate tests, which evaluate the components of $\hat{\tau}_{\mathrm{i}}$ and $\hat{\alpha}_{\mathrm{i}}$ individually (e.g., ANOVA) are not invariant. Since one will usually want to follow a significant MANOVA with a series of univariate tests to discover which of the individual coefficients are contributing to the overall difference, it is important to realize that the results of the univariate tests depend on whether $\mathrm{W}$ or $\Phi$ was used. Moreover, the results can differ qualitatively as well as quantitatively: We have encountered a number of situations in which "more satisfactory" results were obtained when $\Phi$ was used in place of $W$. It is difficult to address this phenomenon in general terms but, e.g., when we reanalyzed Allen's (1983) data set the multivariate (using all the $\hat{\tau}_{i}$ 's or all the $\hat{\alpha}_{i}$ 's) tests for group differences were highly significant; yet none of the group comparisons based on the $\hat{\tau}_{i}$ 's individually were even close to significant. The univariate tests based on the elements of the $\dot{\alpha}_{i}$ 's were more in line with natural expectation. Similar results were subsequently obtained by using a number of other data sets; the details for Allen's (1983) data are provided later.

We should also note here that, while it is not always possible to decide on which is "more satisfactory," differing results can be expected in other longitudinal data analysis contexts as well. For example, the choice of which covariates should be used in Rao's (1965a) procedure depends on the correlations between the regression coefficients actually used to model the data and the higherorder coefficients whose inclusion was not required to achieve an acceptable fit. These correlations can differ markedly, and need not even have the same sign, as can be seen by comparing Goldstein (1979), who used W, with Rao (1965a) and/or Grizzle and Allen (1969), who used $\Phi$ on the same data set. When $W$ is used, the correlation between two regression coefficients depends on the degree of the polynomial fit to the data, e.g., the correlation between the coefficients of $t$ and $\mathrm{t}^{5}$ are in general different for $\mathrm{D}=2$ and 
$\mathrm{D}=3$. This somewhat counterintuitive situation can be avoided by using $\Phi$ instead of $W$.

$V$. Rao (1965b) showed that the use of $\Phi$ was optimal (in the sense of minimizing the variances of the estimators of the regression coefficients under mild restrictions on the choice of the design matrix) in ordinary least squares regression problems. That is, if $E(x)=W \tau$ and $V(x)=\Sigma=\sigma^{2} I$, then given $W$ satisfying $W_{i}^{\prime} W_{i}=C_{i}^{2}$, where $W_{i}$ is the $i^{t h}$ column of $W$ and $C_{i}$ a constant, $V(\hat{\tau})=\left(\mathbf{W}^{\prime} \mathbf{W}\right)^{-1} \sigma^{2}$ is minimized when $\mathrm{W}_{\mathrm{i}}{ }^{\prime} \mathrm{W}_{\mathrm{j}}=0$, i.e., when the columns of $\mathbf{W}$ are orthogonal, viz., when $\Phi$ is used. The condition that $\mathrm{W}_{\mathrm{i}}{ }^{\prime} \mathrm{W}_{\mathrm{i}}=\mathrm{C}_{\mathrm{i}}{ }^{2}$ can be interpreted in the context of restricting the model $E(x)=W_{T}$ to a range of values of $t$ for which the model is presumed valid (Rao, 1965b).

Since in the two-stage model $V(\hat{\tau})=\Lambda+\left(W^{\prime} W\right)^{-1} \sigma^{2}$, Rao's result applies and $V(\hat{\alpha}) \leqslant V(\hat{\tau})$. Thus, in the two-stage model, one of the models for which (5) is used, more efficient estimators will result when $\Phi$ is used in place of $W$.

This result can also be phrased in terms of variance inflation factors (e.g., Neter et al., 1985). These factors measure how much the variances of the estimated regression coefficients are inflated when nonzero correlations between the independent variables (columns of $\mathbf{W}$ ) exist. The estimated regression coefficients will tend to have relatively large sampling variability when the columns of $\mathrm{W}$ are highly correlated. For example, for the $10 \times 4$ matrix $W$ of the form (7) with $\mathrm{t}=1,2, \ldots, 10$ and $\mathrm{D}=3, \mathrm{r}_{23}=0.975$, $r_{24}=0.882$, and $r_{34}=0.961$, where $r_{i j}$ is the correlation between the $\mathrm{i}^{\text {th }}$ and $\mathrm{j}^{\text {th }}$ columns of $\mathrm{W}$. For the $5 \times 3 \mathrm{~W}$ with $\mathrm{t}=1,2,3,4,5$ and $\mathrm{D}=2, \mathrm{r}_{23}=0.981$. These correlations are typical of those obtained in practical situations when $W$ is of the form (7). When $\Phi$ is used, the correlations are, of course, zero. It would appear, then, that the sampling variabilities of the regression coefficients when $\mathrm{W}$ is used will be substantially higher than those obtained when $\Phi$ is employed.

VI. Nothing is lost by using OPRC's. While we have some sympathy for Goldstein's $(1979$, p. 93) remark that the f's have a "more convenient interpretation," one can always recover the $\hat{\tau}$ 's from the $\hat{\alpha}$ 's via the equation

$$
\hat{\tau}_{\mathbf{i}}=\mathbf{W}^{-} \Phi \hat{\alpha}_{\mathbf{i}}
$$

where $\mathbf{W}^{-}$is a generalized inverse (Graybill, 1969) of W. This yields a unique solution for $\hat{\tau}_{i}$ provided only that $\operatorname{rank}(\mathrm{W})=\mathrm{P}$ (Graybill, 1969), one of the standard constraints on the time design matrix. (Note that, without this constraint, $\hat{\tau}$ itself would not be uniquely determined.) But it should be also realized that the $\hat{\tau}$ 's are not always easier to interpret. An example which will be familiar to many readers should suffice to illustrate this point. In the mandibular ramus height data set analyzed by, among others, Elston and Grizzle (1962), Grizzle and Allen (1969), Rao (1965a), and Goldstein (1979), 20 boys were measured at $8,8.5,9$, and 9.5 years of age. The mean values at these time points were $48.66,49.62,50.57$, and 51.45 , respectively. A line was found to fit the data adequately. If

$$
\mathrm{W}=\left[\begin{array}{cc}
1 & 8 \\
1 & 8.5 \\
1 & 9 \\
1 & 9.5
\end{array}\right]
$$

is used to produce $x(t)=\hat{\tau}_{1}+\hat{\tau}_{2} t$, then $\hat{\tau}_{2}=1.87$ is the rate per half-year, but $\hat{\tau}_{1}=33.75$ is the (estimated) mean value at $t=0$. This may be "easy to interpret," but it is not even a proper object of inference given these data. There are no observations in the region around $t=0$ and there is no guarantee that a line will even be appropriate there. It would be difficult to interpret any differences which might be found between groups based on $\hat{\tau}_{1}$. If, on the other hand, one were to use

$$
\Phi=\left[\begin{array}{rr}
1 & -3 \\
1 & -1 \\
1 & 1 \\
1 & 3
\end{array}\right]
$$

$x(t)$ would have the form $x(t)=$ $\hat{\alpha}_{0}+\hat{\alpha}_{1} \phi_{1}(t)=\hat{\alpha}_{0}+\hat{\alpha}_{1}(2 t)$. In the example, $\hat{\alpha}_{1}=0.4655$ is the rate per quarter-year and $\hat{\alpha}_{0}=50.075$ is the (estimated) mean value at $\mathrm{t}=8.75$ years.

It would appear, then, that many investigators will want to use OPRC's in certain situations. Accordingly, a GAUSS program was written, which, given $X$ and $t_{1}, t_{2}, \ldots$, $\mathrm{t}_{\mathrm{T}}$, computes $\hat{\alpha}$ and $\hat{\tau}$ and (optionally) saves either or both of these matrices in an ASCII file so that they may be analyzed by using any of our (or others') programs. The user can also print $\Phi$ in either standardized $\left(\Phi^{\prime} \Phi=I\right)$ or unstandardized ( $\Phi^{\prime} \Phi$ diagonal) 
form. This can be done even when no $\mathrm{X}$ matrix is provided, i.e., one can obtain $\Phi$ from the program instead of consulting tables (e.g., Pearson and Hartley, 1958; Fisher and Yates, 1964) when the t's are equally spaced. The program can also be used for calculating $\Phi$ (following, e.g., Wishart and Metakides, 1953; Robson, 1959) in the unequally spaced case. These will prove useful not only in longitudinal data analytic contexts, but in other situations as well, e.g., ordinary least squares regression and trend analysis (Winer, 1962).

\section{THE PROGRAM}

The GAUSS program, ORPOL, provides the within-individual (time) design matrix and, if a data matrix is input, the corresponding estimated regression coefficients. The program is invoked with the command

\section{GAUSSRUN ORPOL}

Subsequent screen output is also sent to the (automatically created) ASCII file ORPOL.OUT where it may be edited and printed. The program begins by asking whether the user has a data set containing the values of a response variable measured across time (i.e., a $\mathrm{X}$ matrix). This matrix, if it exists, can be either in an ASCII file or in GAUSS data set format, the values of the observations over time occupying successively numbered variables (i.e., a block of columns) in the $\mathrm{X}$ matrix. If the data are in a GAUSS data set, there is no requirement that these variables begin with variable number 1 (e.g., a response measured at four times could be stored in variables 3 through 6). If in an ASCII file, it is assumed that this requirement is satisfied.

If the response is no (i.e., one does not have a X matrix), the user is prompted for the number of time points and the degree of the polynomial and asked whether he/she wishes to enter the values of the time variable. If no, the program uses the integers 1 , $2, \ldots, \mathrm{T}$; if yes, the user is requested to enter the times of measurement, one value per line. Finally, the user selects the form of the $\mathrm{W}$ (or $\Phi$ ) matrix desired from among the possibilities:

1. Noninteger orthogonal scores.

2. Integer orthogonal scores (equally spaced time points only).

3. Orthonormal scores.

4. Original time scores.
The program then prints the time design matrix and saves it in ORPOL.OUT along with all the other output displayed on the screen.

If the user indicates that an $\mathrm{X}$ matrix is available, the program first asks whether $\mathrm{X}$ is in an ASCII file or a GAUSS data set. If the response is ASCII (A), the user is asked for the name of the file (including any extension of the file name) and the values of $\mathrm{N}, \mathrm{T}$, and $D$. If the data are in a GAUSS (G) data set, the user is asked for the name of the data set (excluding any extension), the variable numbers (columns) of the first and last response variables, and the degree of the model to be used. In either case, the user is then asked whether or not he/she wishes to save the estimated regression coefficients (in an automatically created ASCII file called ORPOL.ASC). It then prompts for the following additional information:

1. Whether or not the user wants to supply the values of the time points.

2 . The numerical values of the time points (one per line) if the answer to item 1 is yes (otherwise the program uses $1,2, \ldots, \mathrm{T}$ ).

3 . The form of the $W$ matrix from the four choices listed previously.

The program then prints the time design matrix $W$ (in the program, $W$ is used to denote both W and all the forms of the $\Phi$ matrix) and the matrix Y of estimated polynomial regression coefficients for each individual in the sample. (Y will consist of either ordinary or orthogonal coefficients depending on the choice of W.) Both $\mathrm{W}$ and $\mathrm{Y}$ are saved in ORPOL.OUT and, if requested $\mathrm{Y}$ is also saved in the ASCII file ORPOL.ASC.

\section{AN EXAMPLE}

Some of the output which can be obtained from the program and the sense in which $\Phi$ produces "more satisfactory" results than W using Allen's (1983) data are illustrated. Allen studied four groups of calves $(n=8$ per group) and analyzed average daily gains in weight $(\mathrm{kg})$ for 7 consecutive 2 -week periods. The time points $1,3,5,7,9,11,13$ (the midpoints of the 2 -week periods) were coded and a $D=6$ degree polynomial was fitted to the data by using the $\mathrm{W}$ form of the time design matrix as in equation (7). MANOVA was used to test for parallelism (all coefficients except the intercept equal in the four groups) and coincidence (all coefficients including the intercept equal), resulting in 
$P=0.0085$ and $P=0.0418$, respectively, using Wilks' likelihood ratio criterion. Finally, the contrasts between groups 1 and 2 , groups 3 and 4 , and the combined groups 1 and 2 vs. 3 and 4 were examined. All comparisons were done on a multivariate basis; Allen did not compare any of the coefficients individually.

We compared the groups on a coefficientby-coefficient basis using our program (Ten Have et al., 1992) for carrying out the Potthoff and Roy (1964) analysis with the arbitrary matrix $\mathbf{A}$ set to $\mathbf{A}=\mathbf{I}$ (this is equivalent to the analysis used by Allen and is one of the situations in which the unweighted estimator of $\tau$ is appropriate). None of the individual coefficients differed significantly in the four groups. While the multivariate tests were highly significant, indicating that the growth curves were not all the same in the four groups, the univariate comparisons which we hoped would tell us "where the significance resided" failed to point to any differences whatsoever. Indeed, none of the $P$ values for the tests of the intercept, linear coefficient, quadratic coefficient, etc., even approaches significance (Table 1).

Allen's data were then reanalyzed by using OPRC's. The multivariate comparisons, as expected, agreed with Allen's results, but when OPRC's were compared individually, the $P$ values shown in Table 2 were obtained. These results do include significant differences for several of the individual OPRC's. This then is the sense in which $\Phi$ produces

TABLE 1. Univariate tests for differences among the four groups for each of the coefficients computed using $\boldsymbol{W}$

\begin{tabular}{ll}
\hline Coefficient & $\frac{P \text { value }}{0.9109}$ \\
$\tau_{1}$ & 0.8777 \\
$\tau_{2}$ & 0.8758 \\
$\tau_{3}$ & 0.9030 \\
$\tau_{4}$ & 0.9344 \\
$\tau_{5}$ & 0.9590 \\
$\tau_{7}$ & 0.9749 \\
\hline
\end{tabular}

TABLE 2. Univariate tests for differences among the four groups for each of the coefficients computed using $W$

\begin{tabular}{ll}
\hline Coefficient & $P$ value \\
\hline$\alpha_{0}$ & 0.08416 \\
$\alpha_{1}$ & 0.02948 \\
$\alpha_{2}$ & 0.02943 \\
$\alpha_{3}$ & 0.04852 \\
$\alpha_{4}$ & 0.3130 \\
$\alpha_{5}$ & 0.2834 \\
$\alpha_{6}$ & 0.9749 \\
\hline
\end{tabular}

"more satisfactory" results than W. When an overall difference exists, it is natural to want to see which of the coefficients are contributing to this difference. In this example, $\Phi$ allows us to accomplish this whereas $W$ produces the paradoxical conclusion that the growth curves are different; yet none of the coefficients defining these curves are. This, it may be noted, is the reverse of the wellknown paradox first pointed out by Rao (cf. Kowalski, 1972) in which univariate tests are significant, but the multivariate test is not. In any event, this example shows that, while the interpretation of the OPRC's themselves may be "less convenient" in some ways there are situations in which their use produces results which are more interpretable. And, while we have not been able to provide a firm theoretical basis for the disparity in results observed above, it should be stressed that the above example is in no way unique. Similar patterns of results were obtained for a number of different data sets, for values of $\mathrm{D}$ less than $\mathrm{T}-1$, and for analysis strategies other than that due to Potthoff and Roy (1964), viz., the two-stage model. We used Allen's data set because it is currently in the literature and we took $\mathrm{D}=\mathrm{T}-1$, following Allen, so that the results of his analysis could be directly compared to our analysis based on OPRC's.

The common thread in the examples considered has been that the variability in the $\hat{\tau}$ 's is much greater than that in the $\hat{\alpha}^{\prime}$ 's. Rao's $(1965 b)$ result guarantees that $V(\hat{\alpha}) \leqslant V(\hat{\tau})$ in the two-stage model. Our experience strongly suggests that this holds in the Potthoff-Roy analysis when $\mathbf{A}=\mathbf{I}$ as well, and that the variances of the $\hat{\alpha}$ 's are considerably smaller than $V(\hat{\tau})$. Some indication of this is provided in Table 3 where the values of the first two coefficients are shown for the first

TABLE 3. Values of the first two coefficients for individual subjects (the first eight) in Allen's data set using $\Phi$ and $W$

\begin{tabular}{ccccc}
\hline & \multicolumn{2}{c}{$\Phi$} & \multicolumn{2}{c}{$\mathrm{W}$} \\
\cline { 2 - 5 } & $\alpha_{0}$ & $\alpha_{1}$ & $\tau_{1}$ & $\tau_{2}$ \\
\hline 1.739 & .3704 & 1.995 & -3.342 \\
& 2.793 & .3307 & -6.025 & 11.33 \\
3.001 & .3666 & -9.256 & 18.04 \\
& 2.770 & .4309 & 1.520 & -1.828 \\
& 2.604 & .6728 & 1.597 & -1.945 \\
& 2.880 & .4120 & 2.217 & -2.632 \\
& 1.957 & .6029 & -0.230 & 0.956 \\
& 2.672 & .4082 & 0.24 & 0.706 \\
Variances & 0.14 & 0.03 & 16.71 & 49.69 \\
\hline
\end{tabular}

The variances were computed using all 32 subjects. 
group in Allen's data set for $\mathrm{W}$ and $\Phi$ with $\Phi^{\prime} \Phi=I$. The variances of these coefficients shown in the table are for all $\mathrm{N}=32$ cases; only the first eight values are shown to conserve space.

The example detailed above was intended to illustrate one of the major advantages of $\Phi$ over W. Two example runs of the program itself are given in Appendices B and C. The first (Appendix B), without an X matrix, shows how to obtain the $\mathrm{W}$ form of the time design matrix for Allen's data set when $\mathrm{D}=6$ for the $\mathrm{T}=7$ equally spaced time points $1,3,5,7,9,11,13$. To obtain the integer-valued orthogonal time scores for this situation, which have been tabulated for equally spaced time points, e.g., by Pearson and Hartley (1958), the user should choose not to enter the time points (i.e., choose the default $t=1,2,3,4,5,6,7$ ) and the second form of the time design matrix. The result is shown in Table 4. These are the values of the orthogonal polynomials (the coefficients are specific to $\mathrm{T}=7$ )

$$
\begin{gathered}
\phi_{0}(\mathrm{t})=1 \\
\phi_{1}(\mathrm{t})=\mathrm{t} \\
\phi_{2}(\mathrm{t})=\mathrm{t}^{2}-4 \\
\phi_{3}(\mathbf{t})=\left(\mathrm{t}^{3}-7 \mathrm{t}\right) / 6 \\
\text { etc. }
\end{gathered}
$$

at the $\mathrm{T}=7$ centered time points $\mathrm{t}=-3$, $-2,-1,0,1,2,3$. Our program prints $\phi_{0}(t)$, but this is not usually included in the published tables (e.g., Pearson and Hartley, 1958) since, for every $t, \phi_{0}(t)=1$. A word of caution concerning notation: It is customary to denote the orthogonal polynomials by $\phi_{0}$, $\phi_{1}, \ldots, \phi_{\mathrm{D}}$ with respective regression coefficients $\alpha_{0}, \alpha_{1}, \ldots, \alpha_{\mathrm{D}}$ and we have followed that convention here. While the $\tau^{\prime}$ 's are numbered $\tau_{1}, \tau_{2}, \ldots, \tau_{P}$ the OPRC's are numbered $\alpha_{0}, \alpha_{1}, \ldots, \alpha_{D}$. If, e.g., a quadratic equation is to be fit to the data, the $\mathrm{W}$ form of the equation is

$$
\mathrm{x}(\mathrm{t})=\tau_{1}+\tau_{2} \mathrm{t}+\tau_{3} \mathrm{t}^{2}
$$

$T A B L E$ 4. Integer-valued $\Phi$ matrix for $T=7$ equally spaced time points

\begin{tabular}{rrrrrrr}
\hline$\phi_{0}$ & $\phi_{1}$ & $\phi_{2}$ & $\phi_{3}$ & $\phi_{4}$ & $\phi_{5}$ & $\phi_{6}$ \\
\hline 1 & -3 & 5 & -1 & 3 & -1 & 1 \\
1 & -2 & 0 & 1 & -7 & 4 & -6 \\
1 & -1 & -3 & 1 & 1 & -5 & 15 \\
1 & 0 & -4 & 0 & 6 & 0 & -20 \\
1 & 1 & -3 & -1 & 1 & 5 & 15 \\
1 & 2 & 0 & -1 & -7 & -4 & -6 \\
1 & 3 & 5 & 1 & 3 & 1 & 1 \\
\hline
\end{tabular}

while the $\Phi$ form is

$$
x(t)=\alpha_{0}+\alpha_{1} t+\alpha_{2}\left(t^{2}-4\right) .
$$

We repeat that to obtain Table 4 , the user should request that the program use the integers $1,2, \ldots, T$ for the values of the time variable, i.e., to respond "no" when asked whether he/she wishes to enter these values. This "quirk" in the program is all but mandated by the way in which the PearsonHartley tables are constructed. The full observational range is taken as $t=1,2, \ldots$, $\mathrm{T}$ and the entries in the table represent the values of the orthogonal polynomials at the centered time points $t-(T+1) / 2$. While our program will "work" for any set of time points separated by one unit, the first $T$ integers are the easiest to obtain and their use is recommended. If the user were to enter the time points $1,3,5,7,9,11,13$ (the actual values used by Allen) the result would not be Table 4 , but rather Table 4 with the entries of the last six columns multiplied by two; e.g., the entries under $\phi_{1}$ would be $-6,-4,-2$, etc. This matrix is orthogonal, but the OPRC's are not as simple as those shown in Table 4. We therefore recommend that when the user wishes to obtain integer-valued orthogonal time scores, he/she employ the first $\mathrm{T}$ positive integers as time points.

The second example run of the program (Appendix C), with an $\mathrm{X}$ matrix, shows how to obtain the orthonormal form of the $\Phi$ matrix and the corresponding OPRC's for the data set considered by Schneiderman and Kowalski (1985, 1989) where a quadratic $(D=2)$ was fit to a measure of mandibular ramus height for $\mathrm{N}=12$ young male rhesus monkeys who were measured at $\mathbf{T}=5$ equally spaced time points. These OPRC's are saved in ORPOL.ASC and thus may be analyzed further by importing them into other programs. This option is for the convenience of the user: The entire session is saved in ORPOL.OUT and the OPRC's are there as well, but they are perhaps more readily usable in ORPOL.ASC. For those with the GAUSS program, $\mathbf{Y}$ (and $\mathbf{W}$ ) is also available for immediate manipulation with GAUSS.

It will be seen from Appendices B and C that the program is completely interactive. The user is prompted for all the information needed to produce the output described above. The entire run of the program is reproduced (from ORPOL.OUT); user responses are enclosed in braces, viz., " \{\} ". 


\section{DISCUSSION}

Human biologists are often faced with the challenge of analyzing longitudinal data sets. In those situations in which polynomial modelling of the growth process is deemed appropriate, and in which the unweighted form of the estimator of the polynomial regression coefficients may be used, the choice of a time design matrix must still be made, viz., shall $W$ or $\Phi$ be used? Starting with the realization that, at least in certain circumstances, the use of $W$ produced paradoxical results, we focused on the alternative choice, $\Phi$, and found it to have a number of distinct advantages. While some of these features may be relatively minor, others assume apparently important roles in producing consistent, "more satisfactory" results.

The case in favor of $\Phi$ was presented and provided a GAUSS program designed to facilitate the use of OPRC's in longitudinal data analysis and to allow direct comparisons between $\mathrm{W}$ and $\Phi$. While experience has suggested that $\Phi$ produces "more satisfactory" results, it is important to put this notion to further empirical testing and it is in this spirit that our program is offered. In any case, the program can be used to completely reproduce the published tables of orthogonal polynomials (which pertain only to equally spaced time points) and to extend the availability of these polynomials to the unequally spaced case. While this paper focused on longitudinal data analysis, orthogonal polynomials have a number of other uses (Draper and Smith, 1966) and the program can be used to facilitate such usage.

Finally, the reader will have noted that he/she can choose between three forms of $\Phi$ in the program, viz.,

\section{Noninteger orthogonal scores.}

2. Integer orthogonal scores.

3. Orthonormal scores.

The first was included mainly for the sake of completeness. While 1 has no advantages over 3, some authors (e.g., Draper and Smith, 1966) present examples wherein $\Phi^{\prime} \Phi$ is diagonal (not the identity). We allow this choice for the convenience of users wishing to work through such examples. We envision that option 2 will be invoked only in those situations where the user wishes to use the program instead of published tables. Remember that this option is available only when the distance between each of the time points is unity (in which case we suggest using $1,2, \ldots$, T). For longitudinal data analysis, option 3 will usually be the best choice for $\Phi$. This parametrization is prevalent in the current literature and there are no restrictions on the time points. In short, the primary purpose of ORPOL is to produce $\Phi$ as in 3 and the associated OPRC's. One may also wish to obtain $\mathrm{W}$ (original time scores) for comparative purposes.

\section{ACKNOWLEDGMENTS}

This research was supported by grant DE08730 from the National Institute of Dental Research.

\section{LITERATURE CITED}

Allen OB (1983) A guide to the analysis of growth curve data with special reference to SAS. Comput. Biomed. Res. 16:101-115.

Bellman R (1970) Introduction to Matrix Analysis. New York: McGraw-Hill.

Bock RD (1979) Univariate and multivariate analysis of variance of time-structured data. In JR Nesselroade and PB Baltes (eds.): Longitudinal Research in the Study of Behavior and Development. New York: Academic Press, pp. 199-231.

Bright JW, Dawkins GS (1965) Some aspects of curve fitting using orthogonal polynomials. Ind. Eng. Chem. Fundam. 4:93-97.

Chinchilli VM, Carter WH (1984) A likelihood ratio test for a patterned covariance matrix in a multivariate growth-curve model. Biometrics 40:151-156.

Church A, Jr. (1966) Analysis of data when the response is a curve. Technometrics 8:229-246.

Das Gupta S, Perlman MD (1974) Power of the noncentral F-test: Effect of additional variables on Hotelling's T test. J. Am. Stat. Assoc. 69:174-180.

Dawson DV. Todorov AB. Elston RC (1980) Confidence bands for the growth of head circumference in achondroplastic children in the first year of life. Am. J. Med. Genet. 7:529-536.

Draper N, Smith H (1966) Applied Regression Analysis. New York: Wiley.

Elston RC, Grizzle JE (1962) Estimation of time-response curves and their confidence bands. Biometrics 18:148-159.

Fearn T (1975) A Bayesian approach to growth curves. Biometrika 62:89-100.

Fearn $\Gamma$ (1977) A two-stage model for growth curves which leads to Rao's covariance adjusted estimators. Biometrika 64:141-143.

Fisher RA, Yates F (1964) Statistical Tables for Biological, Agricultural and Medical Research. New York: Hafner.

Goldstein H (1979) The Design and Analysis of Longitudinal Studies. New York: Academic Press.

Graybill FA (1969) Introduction to Matrices With Applications in Statistics. Belmont, CA: Wadsworth.

Grizzle JE, Allen DM (1969) Analysis of growth and response curves. Biometrics 25:307-318.

Guire KE, Kowalski CJ (1979) Mathematical description and representation of developmental change functions on the intra- and interindividual levels. In. JR Nesselroade and PB Baltes (eds.): Longitudinal Research in 
the Study of Behavior and Development. New York: Academic Press, pp. 89-110.

Kowalski CJ (1972) A commentary on the use of multivariate statistical methods in anthropometric research. Am. J. Phys. Anthropol. 36:119-131.

Kowalski CJ, Guire KE (1974) Longitudinal data analysis. Growth 38:131-169.

Marubini E, Milani S (1986) Approaches to the analysis of longitudinal data. In F Falkner and J Tanner (eds.); Human Growth, Vol. 3. New York: Plenum, pp. 79-94.

McMahan CA (1981) An index of tracking. Biometrics $37: 447-455$.

Morrison DF (1972) The analysis of a single sample of repeated measurements. Biometrics 28:55-71.

Neter J, Wasserman W, Kutner MH (1985) Applied Linear Statistical Models. Homewood, IL: Irwin.

Pearson ES, Hartley HO (1958) Biometrika Tables for Statisticians. Cambridge: Cambridge University Press.

Potthoff RF, Roy SN (1964) A generalized multivariate analysis of variance model useful especially for growth curve problems. Biometrika 51:313-326.

Rao CR (1958) Some statistical methods for the composition of growth curves. Biometrics 14:1-17.

Ran CR (1959) Some problems involving linear hypotheses in multivariate analysis. Biometrika 46:49-58.

Rao CR (1965a) The theory of least squares when the parameters are stochastic and its application to the analysis of growth curves. Biometrika 52:447-458.

Rao CR (1965b) Linear Statistical Inference and Its Applications. New York: Wiley.

Robson DS (1959) A simple method for constructing orthogonal polynomials when the independent variable is unequally spaced. Biometrics 15:187-191.

Schneiderman ED, Kowalski CJ (1985) Implementation of Rao's one-sample polynomial growth curve model using SAS. Am. J. Phys. Anthropol. 67:323-333.
Schneiderman ED, Kowalski CJ (1989) Implementation of Hills' growth curve analysis for unequal-time intervals using GAUSS. Am. J. Hum. Biol. 1:31-42.

Schneiderman ED, Kowalski CJ, Ten Have TR (1990) A Gauss program for computing an index of tracking from longitudinal observations. Am. J. Hum. Binl $2: 475-490$.

Stewart GW (1985) Matrix, ill-conditioned. In S Kotz, NL Johnson, and CB Read (eds.): Encyclopedia of Statistical Sciences, Vol. 5. New York: Wiley, pp. 325-326.

Ten Have TR, Kowalski CJ, Schneiderman ED (1991) A program for analyzing one-sample longitudinal data sets which satisfy the two-stage polynomial growth curve model. Am. J. Hum. Biol. 3:269-279.

Ten Have TR, Kowalski CJ, Schneiderman ED, Willis SM (1992) Two programs for performing multigroup longitudinal data analyses. AJPA. Am. J. Phys. Anthropol. (in press).

Ware JH (1983) Growth curves. In S Kotz, NL Johnson, and CB Read (eds.): Encyclopedia of Statistical Sciences, Vol. 3. New York: Wiley, pp. 539-542.

Ware JH, Wu MC (1981) Tracking: Prediction of future values from serial measurements. Biometrics $37: 427-437$.

Winer BJ (1962) Statistical Principles in Experimental Design. New York: McGraw-Hill.

Wishart $\mathbf{J}$ (1938) Growth rate determinations in nutrition studies with the bacon pig, and their analysis. Biometrika $30: 16-28$.

Wishart J, Metakides (1953) Orthogonal polynonial fitting. Biometrika 40:361-369.

Zerbe GO (1979) Randomization analysis of the completely randomized design extended to growth and response curves. J. Am. Stat. Assoc. 74:215-221.

Zerbe GO, Walker SH (1977) A randomization test for comparison of groups of growth curves with different polynomial design matrices. Biometrics 33:653-657.

resident programs such as Windows. No additional software is required (other than what one would normally use to enter a data set); run-time modules and associated files are supplied with the program so that no compiler or interpreter is necessary. The program, written in GAUSS, version 2.0, revision 20 , requires no additional installation or modification and is run with a single command. When requesting the program, address inquiries to EDS and make checks payable to Baylor College of Dentistry. 
APPENDIX B: EXAMPLE RUN WITH NO X MATRIX

Estimation of orthogonal polynomial models (Orpol)

by E.D. Schneiderman, C.J. Kowalski and T.R. Ten Have

Date: $6 / 1 / 90$

Time: $9: 19: 27$

Output is going to ORPOL.OUT

Do you have a Gauss or ASCII dataset containing a response variable measured across time? (Enter ' $\mathrm{Y}$ ' or ' $\mathrm{N}$ ')

(If no, the program will only generate the time design matrix) $\{\mathrm{n}\}$

Enter number of time points: $\{7\}$

Enter degree of model: $\{6\}$

Do you want to enter the values of the time variable? (Enter ' $\mathrm{Y}$ ' or 'N')

$\{y\}$

(If no, the program will provide consecutive integers beginning with one for the time variable)

Enter values of time variable (one value per line)

1

3

5

7

9

11

13

For the time design matrix $(W)$,

Enter: 1 if non-integer orthogonal scores

2 if integer orthogonal scores for evenly spaced time points

3 if orthonormal scores

$\{4\}$

4 if original time scores

Time design matrix based on original time scores

$\begin{array}{rrrrrrr}1.0000 & 1.0000 & 1.0000 & 1.0000 & 1.0000 & 1.0000 & 1.0000 \\ 1.0000 & 3.0000 & 9.0000 & 27.000 & 81.000 & 243.00 & 729.00 \\ 1.0000 & 5.0000 & 25.000 & 125.00 & 625.00 & 3125.0 & 15625 . \\ 1.0000 & 7.0000 & 49.000 & 343.00 & 2401.0 & 16807 . & 117650 . \\ 1.0000 & 9.0000 & 81.000 & 729.00 & 6561.0 & 59049 . & 531440 . \\ 1.0000 & 11.000 & 121.00 & 1331.0 & 14641 . & 161050 . & 1.7716 \mathrm{E}+006 \\ 1.0000 & 13.000 & 169.00 & 2197.0 & 28561 . & 371290 . & 4.8268 \mathrm{E}+-006\end{array}$

$\mathrm{W}$ is the $7 \times 7$ time design matrix 
APPENDIX C: EXAMPLE RUN WITH AN X MATRIX

Estimation of orthogonal polynomial models (Orpol) by E.D. Schneiderman, C.J. Kowalski and T.R. Ten Have

Date: $6 / 1 / 90$

Time: $9: 25: 19$

Output is going to ORPOL.OUT

Do you have a Gauss or ASCII dataset containing a response variable measured across time? (Enter ' $\mathrm{Y}$ ' or ' $\mathrm{N}$ ')

(If no, the program will only generate the time design matrix) $\{\mathrm{y}\}$

Do you want to use a GAUSS or ASCII data set? Enter G or A: $\{\mathrm{g}\}$ *** GAUSS DATA SET INPUT***

NOTE-DATA SET MUST BE A MATRIX WHERE EACH SUBJECT IS A ROW AND EACH VARIABLE (COLUMN) IS A TIME-POINT

THE VARIABLE NUMBERS OF THE RESPONSE VARIABLES MUST BE CONSECUTIVE (E.G. THE 1ST RESPONSE VARIABLE IS

VARIABLE 3, THE 2ND RESPONSE IS VARIABLE 4, ETC.)

YOU MUST KNOW THE VARIABLE (COLUMN) NUMBERS OF THE FIRST AND LAST RESPONSE VARIABLES

DO NOT INCLUDE THE EXTENSION OF THE FILE NAME OF THE GAUSS DATASET. (E.G. ENTER: INDATA, NOT INDATA.DAT)

Enter GAUSS data set containing the response variables (X):

FILE NAME $=\{$ ramus $\}$

Enter column number for first response variable $\{1\}$

Enter column number for last response variable $\{5\}$

Number of time points $=5$

Fnter degree of model: $\{2\}$

Do you wish to save the polynomial regression coefficients in an ASCII file (Enter ' $\mathrm{Y}$ ' or 'N') $\{\mathbf{y}\}$

The polynomial regression coefficients will be saved in ORPOL.ASC

Do you want to enter the values of the time variable? (Enter ' $\mathrm{Y}$ ' or ' $\mathrm{N}$ ')

$\{n\}$

(If no, the program will provide consecutive integers beginning with one for the time variable) 
For the time design matrix $(W)$,

Enter: 1 if non-integer orthogonal scores 2 if integer orthogonal scores for evenly spaced time points 3 if orthonormal scores $\{3\}$ 4 if original time scores

orthonormal time scores
$\begin{array}{lll}0.45 & -0.63 & 0.53\end{array}$
$0.45 \quad-0.32 \quad-0.27$
$0.45 \quad 0.00 \quad-0.53$
$\begin{array}{lll}0.45 & 0.32 & -0.27\end{array}$
$\begin{array}{lll}0.45 & 0.63 & 0.53\end{array}$

$\mathrm{W}$ is the $5 \times 3$ time design matrix

GROWTH CURVE COEFFICIENTS FOR INDIVIDUALS

Press any key to continue

$\begin{array}{llr}71.02 & 8.665 & -2.512 \\ 81.26 & 13.31 & -1.684 \\ 76.03 & 10.28 & -2.806 \\ 83.27 & 14.10 & -3.742 \\ 74.06 & 10.63 & -2.619 \\ 81.88 & 12.97 & -0.9087 \\ 81.66 & 14.14 & -4.036 \\ 83.72 & 13.53 & -2.886 \\ 83.09 & 14.10 & -3.849 \\ 81.17 & 10.25 & -2.138 \\ 80.36 & 13.95 & -0.8820 \\ 82.96 & 14.26 & -4.356\end{array}$

$\mathrm{Y}$ is the $12 \times 3$ matrix that contains the 2 th degree polynomial coefificients

The polynomial regression coefficients have been saved in ORPOL.ASC 